\title{
Early Intervention for Autism With a Parent-Delivered Qigong Massage Program: A Randomized Controlled Trial
}

\author{
Louisa M. T. Silva, Mark Schalock, Kristen Gabrielsen
}

KEY WORDS

- autistic disorder

- early intervention (education)

- massage

- parents

- sensory disorders
Louisa M. T. Silva, MD, MPH, is Visiting Professor, Teaching Research Institute, Western Oregon University, P.0. Box 688, Salem, OR 97308; Imtsilvaqigong@comcast.net

Mark Schalock is Associate Research Professor, Teaching Research Institute, Western Oregon University, Monmouth.

Kristen Gabrielsen, MPH, is Consultant/Project Manager, Teaching Research Institute, Western Oregon University, Monmouth.
A recent randomized controlled trial (RCT) of a dual parent and trainer-delivered qigong massage intervention for young children with autism resulted in improvement of measures of autism as well as improvement of abnormal sensory responses and self-regulation. The RCT evaluated the effects of the parent-delivered component of the intervention. Forty-seven children were randomly assigned to treatment and wait-list control groups. Treatment group children received the parent-delivered program for 4 mo. Trained therapists provided parent training and support. Improvement was evaluated in two settings — preschool and home-by teachers (blind to group) and parents. Results showed that the parent-delivered program was effective in improving measures of autism (medium effect size) and sensory and self-regulatory responses (large effect size). Teacher data on measures of autism were confirmed by parent data. Results indicate that the parentdelivered component of the program provided effective early intervention for autism that was suitable for delivery at home.

Silva, L. M. T., Schalock, M., \& Gabrielsen, K. (2011). Early intervention for autism with a parent-delivered qigong massage program: A randomized controlled trial. American Journal of Occupational Therapy, 65, 550-559. doi: 10.5014/ ajot.2011.000661

7 he rising incidence of autism, in concert with growing public awareness and progress in early diagnosis, has resulted in many more children being identified in the preschool period (Interagency Autism Coordinating Committee, 2011) and referred for early intervention than in the past. This situation creates a window of opportunity for early intervention programs to remediate the social and language delays characteristic of autism and to prepare children for primary school. To date, several early intervention approaches have been developed for preschool populations with varying degrees of success and drawbacks that include high cost and unavailability of services (Ospina et al., 2008).

A recent randomized controlled trial (RCT) of a 5-mo qigong (pronounced "chee-gong") treatment for preschool children with autism known as Qigong Sensory Training (QST) showed significant improvement in behavioral, social, and language measures of autism in treated children as well as improvement in sensory impairment and self-regulation (Silva, Schalock, Ayres, Bunse, \& Budden, 2009). The QST treatment is a manual therapy based on principles of Chinese medicine consisting of a protocol of 12 intentional patting, shaking, and pressing movements applied to 12 areas of the body while recipients are clothed. The treatment was delivered by trained occupational therapists and other early intervention staff as well as by the children's parents. It was directed at improving sensory and self-regulatory impairment (Silva, Ayres, \& Schalock, in press) and resulted in a calmer child who was more aware and better able to participate socially. A trained staff person gave the child a skilled version of the treatment 20 times over 5 mo, whereas parents were taught a simpler version that was given daily. The parent version was designed to maintain balance in the 
child and consolidate the gains on a daily basis. The skilled version was designed to advance the progress of the child.

The 5-mo, 80-hr training for staff to competently deliver the skilled version of the intervention has been described in a previous article (Silva, Ayres, \& Schalock, 2008). The training includes basic concepts of Chinese medicine as applied to autism as well as manual skills in working with the wide variety of sensory presentations seen in children on the autism spectrum. By contrast, the parent training is shorter and concentrates on the manual techniques as applied to the individual child. Parents receive an initial 3-hr training, which is augmented with additional coaching during subsequent treatment visits by the trained staff.

Notwithstanding the importance of the results obtained with this intervention, a drawback to its dissemination into state-sponsored early intervention programs in Oregon is that the service time required for the staff component of this intervention-20 half-hour visits over 5 mo-is more than is normally allotted for autism services delivered by occupational therapists. To make the intervention accessible to local early intervention programs, our research team was asked by rural program administrators to develop a parent-delivered intervention that could be supervised by trained staff without requiring direct treatment; the intervention could then be included under the service rubric of parent training. This request coincided with our own desire to optimize the parent-delivered component of the intervention and to conduct a pilot study to evaluate its effect on treatment outcome.

Published research on parent-delivered programs for autism has shown successful outcomes when parents are given adequate training and support, although in some cases less success than when the intervention is delivered by professional staff (Barlow, Powell, Gilchrist, \& Fotiadou, 2008; Diggle, McConachie, \& Randle, 2003; McConachie $\&$ Diggle, 2007). Our experience indicated that the success of the parent component of the intervention depended on parents' being able to incorporate the QST treatment into the child's routine and to follow through with it on a daily basis. When children received consistent, daily treatment, the goals of improved sensory and self-regulation, as well as improved overall learning and behavior, were achieved. Several barriers must be overcome to reach those goals, including the child's refusal of touch on different parts of his or her body, the parent's unfamiliarity with the principles on which QST treatment is based, the parent's unfamiliarity with the healing process that the treatment initiates and drives, and the parent's commitment to the necessity of a daily intervention.
To design a parent training and support program that would provide parents of different educational and cultural backgrounds with the greatest chance of success with the intervention, we first reflected on our $9 \mathrm{yr}$ of experience in teaching parents how to give the intervention. For the 3 preceding yr, we studied the language that parents used to describe what the treatment did for their child, and we turned to this language to develop a curriculum for parents that could communicate the Chinese medicine concepts important to the success of QST. If children were not going to receive the skilled trainer component of the intervention, we would need to teach parents the skills that we had previously transmitted to trainers.

On the basis of these and other considerations, a training and support program for parents was designed and piloted. This program was called the QST Home Program. As with our previous research, we planned to recruit study participants from children receiving autism services in local, state-sponsored early intervention preschool programs. The efficacy of the Home Program would be evaluated against a wait-list control group using an RCT design. Consistent with our previous research, we would also examine the correlation between changes in sensory and self-regulation and changes in autistic behavior.

Subsequently, we planned to treat the wait-list control group with the Home Program and compare the pooled outcomes of the Home Program with previously pooled data from the dual intervention. It had been our impression that the additional time and support that the dual intervention afforded families was important to a successful outcome when (1) children were more severely affected and (2) parents were more severely stressed by the autism. Should differences be identified, the information might guide future research relative to determining the optimal intensity of intervention within a limited resources context.

\section{Research Questions}

1. Do children receiving the QST Home Program improve in the classroom setting on measures of autistic behavior as evaluated by preschool teachers blind to group? Do they improve in the home setting on measures of autism and sensory and self-regulation as reported by their parents?

2. Do children in the Home Program and the Dual Program experience equivalent outcomes on measures of autism and sensory and self-regulation?

3. Are there differences in outcomes between the Dual and Home Program interventions relative to the severity of autism and the severity of parent stress? 
Method

\section{Participants}

Recruitment was conducted by sending an invitation letter to parents of children ages 3-6 yr receiving autism services from state-funded, early intervention programs in the Salem and Portland areas in Oregon. Criteria for entry into the study included age $<6$ years; receiving early intervention services for autism; and no complicating medical diagnoses or chronic medication, including no active medical therapies for autism such as chelation. Most children attended early intervention preschools 5-10 hr per week. Mean age was 58 mo. Parents agreed not to begin additional interventions for autism during the study, to transport their children to the training and support visits, and to give their child a daily QST treatment for the duration of the study.

Children were randomly assigned to treatment and wait-list control conditions using a random number generator. The study was carried out with Western Oregon University Institutional Review Board approval.

\section{QST Parent Training and Support Program}

Parent training began with a 3-hr group training that was attended by one or both parents or caregivers and the trainer who would be working with them. The research and reasoning behind the intervention was explained, and then parents and caregivers practiced the 12 parts of the treatment on each other under the guidance of a trainer until they could do each movement correctly. They were also given an introduction to the predictable changes that children experience in response to treatment and how to modify their manual technique and parenting approach accordingly. In addition, they were given a booklet, a DVD, and a chart explaining QST and covering the didactic material given in the 3 -hr training (Silva, 2008).

After the 3-hr group training, the coaching and support program was delivered in 7 weekly, 30-min support meetings at which parents and caregivers brought the child to the clinic to meet with the trainer. During these support meetings, the trainer observed and coached the parent giving the massage and evaluated and recorded fidelity to the QST procedures. During the first half of this study, trainers reported that all parents had incorporated the treatment into the child's daily routine and were providing it correctly. During the second half of the study, two families did not continue to give the daily treatment after the last coaching session. All trainers participating in the research program had previously graduated from the 80-hr QST skill-based curriculum (Silva et al., 2008).

\section{Treatment Protocol}

The QST protocol takes approximately $15 \mathrm{~min}$ for the parents to deliver and consists of a sequence of 12 patting, shaking, or pressing movements modified according to the physical responses of the child as described in the manual. (The QST Home Program training and support program for parents is described in full in a parent training manual and DVD titled "Qigong Massage for Your Child With Autism: A Home Program From Chinese Medicine" [Silva, 2011].)

The movements have the following actions on the body according to Chinese medicine:

- Movements 1, 2, 3: Open up and promote the circulation to the brain and senses.

- Movement 4: Clear additional functional impediments of the ear.

- Movements 5, 6, 7: Promote social interaction, speech, and self-regulation.

- Movements 8, 9: Strengthen digestion and elimination; strengthen physical vitality.

- Movements 10, 11, 12: Calm the child and improve sleep.

\section{Evaluation Instruments}

The Autism Behavior Checklist (ABC; Krug, Arick, \& Almond, 1980, 1993) was chosen to evaluate autistic behavior in the classroom and was completed by preschool classroom teachers who were blind to the treatment and control groups. The ABC measures behaviors typical of autism in five domains: sensory, relating, body and object use, language, and social and self-help. It is part of the Autism Screening Instrument for Educational Planning 3 (Krug, Arick, \& Almond, 2003), an autism evaluation tool commonly used for evaluation and planning for children with autism spectrum disorders, and is well known to the special education teachers who participated in this study. The ABC provides raw scores ranging from 0 to 167 . A score of $\geq 54$ is consistent with autism. Eaves and Williams (2006) reported an $\alpha$ coefficient of .89 .

The parent version of the Pervasive Developmental Disorders Behavior Inventory (PDDBI; Cohen \& Sudhalter, 2005) was used to measure social and language abilities and maladaptive behavior. High levels of internal consistency have been reported ( $\alpha$ s $=.80-.98$ across domains and constructs). The PDDBI generated an autism composite score that was a composite measure of autism severity in the home setting. The Sense and SelfRegulation Checklist (SSC; Silva \& Schalock, in press) 
was developed to measure sensory and self-regulatory symptoms commonly reported by parents. Sense scores range from 0 to 75 , and self-regulation scores range from 0 to 99 . This instrument has demonstrated relatively high levels of internal consistency $(\alpha=.87)$. The Autism Parenting Stress Index (APSI; Silva \& Schalock, 2011) was developed to measure parent stress in 13 aspects of autism of concern to parents. Current validation data indicate reasonable levels of internal consistency $(\alpha=.83)$.

\section{Statistical Analysis}

Pretest-posttest paired $t$ tests were conducted for all treatment and control groups to determine whether, over the course of the study, there were any significant gains (either statistical and practical) in the treatment and control groups. After these initial $t$ tests, we approached data analysis somewhat differently for each of the three hypotheses tested.

Preassessment scores for intervention and control groups were analyzed to determine equivalence. This information was important in determining the appropriate analyses for testing the main hypotheses of the study. Independent $t$ tests were conducted on measures of autistic behavior and parental stress. Multiple analysis of variance (MANOVA) was used separately for the PDDBI and SSC. Post hoc Bonferroni-adjusted individual $t$ tests for independent samples were conducted to further test group equivalence on the preassessment outcome measures to document more precisely any differences that might exist on more specific impairments and abilities.

If indicated, a series of analysis of covariance (ANCOVA) and multivariate analysis of covariance (MANCOVA) analyses with Bonferroni adjusted post hoc analyses were conducted to determine the significance (both statistical and practical) of any treatment effects. These analyses were conducted separately on the various measures using postassessment scores as the dependent variables, group as the independent variable, and preassessment scores as covariates. SPSS (Version 18; IBM Corp., Somers, NY) generates partial $\eta^{2}$ as an effect size estimate in the General Linear Model (Haase, 1983; Tabachnick \& Fidell, 1989). Partial $\eta^{2}$ is equivalent to $R^{2}$. Using the formula for deriving $r$ from Cohen's $d$ (Hedges, 1982), it is possible to establish ranges in partial $\eta^{2}$ that coincide with Cohen's (1988) original small, medium, and large classifications, with .01-.06 indicating a small effect size, .06-.14 indicating a medium effect size, and $>.14$ indicating a large effect size.

Equivalence testing of Dual and Home Program treatment outcomes was done using the confidence interval (CI) approach (Schuirmann, 1981, 1987). Equivalence testing is useful when the researcher wants to show that two means are not statistically different and requires the identification of a clinically significant difference $(\delta)$ between outcomes of competing treatments. In practice, $\delta$ is often chosen to be a percentage (usually 15\%-25\%) of the mean of the comparison group (Mecklin, 2002). This number is then used to create a tolerance interval around zero beyond which equivalence would be rejected. For this analysis, the dual treatment group was used as the standard of comparison, and the $\delta$ was selected as $15 \%$ of the adjusted mean postassessment score. A unique tolerance interval was constructed for each outcome variable, and the 95\% CI for the difference in adjusted mean scores between Dual Program and Home Program groups was compared with the tolerance interval.

We used Pearson correlation analysis to determine the relationship between sensory regulation impairment and autism. This analysis informed the assessment of whether differential treatment effects might be found in the Dual and Home Programs on the basis of severity of sensory regulation impairment. We used a two-way MANOVA approach with treatment type and a level-of-severity dichotomous variable as factors on PDDBI postassessment scores to determine whether there were differential effects in treatments by initial severity of sensory regulation impairment or severity of parent stress.

\section{Results}

Forty-seven children (33 boys and 14 girls) met eligibility criteria. Of the initial 47 children, 28 children were assigned to the intervention group and 19 children were assigned to the wait-list control group. Uneven groups were the result of multiple sibling pairs within the participants. One family dropped out of the control group (1 child). Two families dropped out of the treatment group (4 children), and two additional families (3 children) dropped out of the second intervention group shortly after enrollment. Subsequently, pretest and posttest data were collected on 24 children in the initial intervention group and 18 children in the wait-list control group. Pretestposttest data were collected on an additional 15 children in the wait-list control group during their intervention condition.

\section{Pre- to Postassessment Changes: Home Program Versus Control Group}

Analyses of pretest and posttest scores indicated significant improvement for treatment group participants. Effect size estimates are mostly in the large range $(\geq .60)$. No significant differences were obtained for wait-list control group participants. These results are shown in Table 1. 


\section{Preassessment Equivalence: Home Program Versus Control Group}

Analysis of the preassessment data for the Home Program treatment and wait-list control group participants completing the study indicated no significant differences in preassessment scores between groups on any of the measures. Independent $t$ tests and Bonferroni-adjusted post hoc $t$ tests within MANOVA generated $t$ statistics ranging from 1.986 to 0.305 with corresponding $p$ values ranging from .057 to .763 . Inspection of the means and standard deviations in the two groups indicate consistently greater variance in the wait-list control group even though means are relatively consistent. The presence of these differences in the data and the deviations from full random assignment indicated ANCOVA and MANCOVA would be preferable to test for main treatment effects and control for initial differences.

\section{Treatment Effects: Home Program Versus Control Group}

Treatment effects varied across measures. Medium effects were found for the teacher measure of autistic behavior, but significant and large effects were found for parent measures of PDDBI Sensory, Behavior, the Autism Composite score, Sensory Impairment, Self-Regulation Impairment, and Parent Stress. Small effects were found for parent measures of Language and Social Abilities. These results are shown in Table 2.

In several cases, MANCOVA analyses produced statistically insignificant $F$ s, yet medium to large partial $\eta^{2}$ effect size estimates, indicating the small sample sizes yielded insufficient power in the analysis. This was the case for the Teacher ABC and the PDDBI.

\section{Pretest-Posttest Gains: Pooled Home Program Versus Pooled Dual Program Treatment Group}

Although the significant and small gains were found with the Home Program intervention on all measures, significant and large gains were found for all measures for children in the Dual Program intervention. In all cases, $p$ values were more significant for the Dual Program intervention. Although effect size estimates were generally larger for the Dual Program treatment, we found some inconsistencies across the two interventions along with inconsistencies in the raw change scores across the interventions. These results can be explained by the differences in standard deviations and sample sizes across treatment groups (Table 3).

\section{Preassessment Equivalence: QST Home Versus Dual Treatment Group}

Analysis of the preassessment data for the Home Program and Dual Program treatment group participants in this study indicated that there were significant differences in preassessment scores between groups on several of the measures. Additional inspection of the means and standard deviations in the two groups indicated inconsistency as well. The presence of these differences in the data and the deviations from full random assignment indicated ANCOVA and MANCOVA were needed to test for differential treatment effects and control for initial differences.

Table 1. Qigong Sensory Training Home Program Treatment and Wait-List Control Group Scores

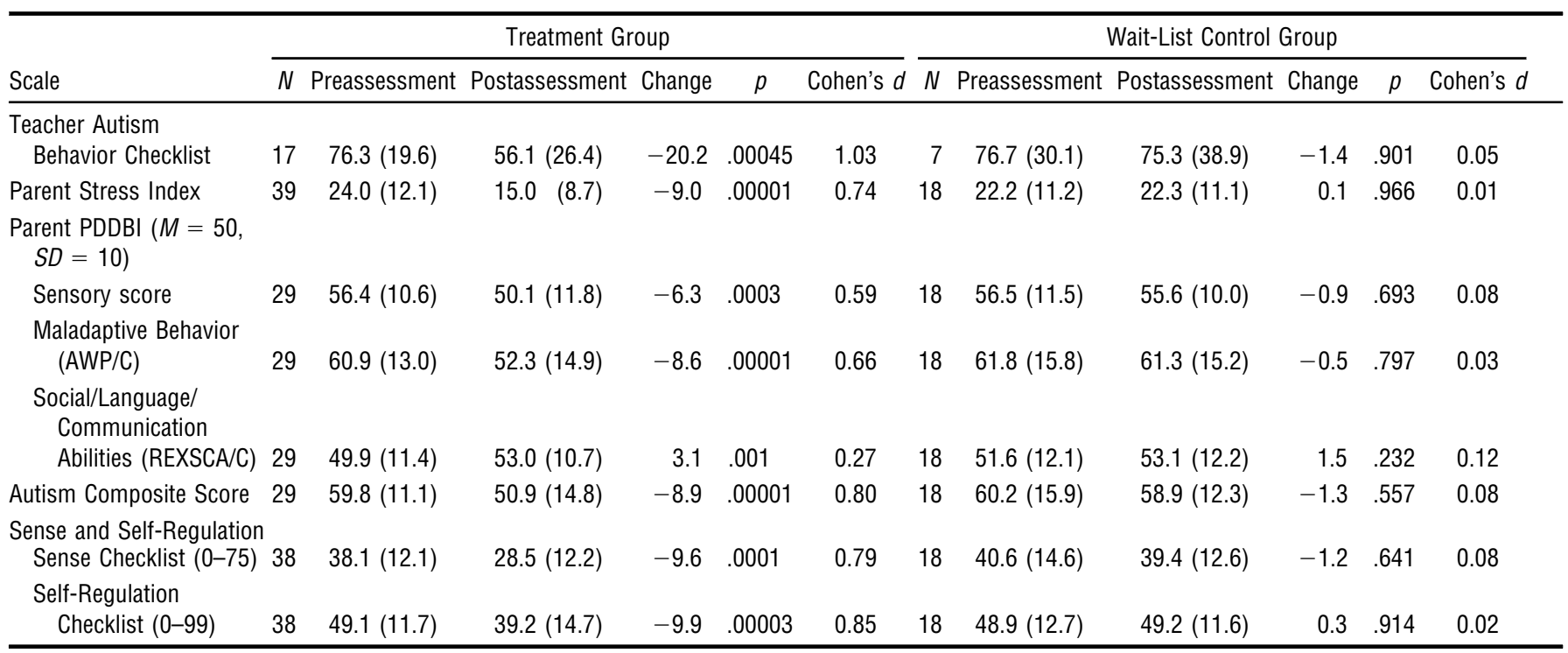

Note. $M=$ mean; $S D=$ standard deviation; PDDBI = Pervasive Developmental Disorders Behavior Inventory; AWP/C = Approach/Withdrawal Problems Composite; REXSCA/C = Receptive/Expressive Social Communication Abilities Composite. 
Table 2. Summary of Analysis of Covariance (ANCOVA) and Multivariate Analysis of Covariance (MANCOVA) Results for Intervention Effects on Measures of Sensory Impairment, Behavior, and Developmental Skills

\begin{tabular}{lrll}
\hline & \multicolumn{3}{c}{ Group Main } \\
\cline { 2 - 4 } Variable & \multicolumn{1}{c}{$F^{\mathrm{a}}$} & $p$ & Partial $\eta^{2 \mathrm{~b}}$ \\
\hline Univariate ANCOVA analyses & & & \\
$\quad$ Autism Behavior Checklist (0-167) & 1.116 & .309 & .074 \\
$\quad$ Parent Stress Index & 15.141 & .0003 & .219 \\
Multivariate analyses: Parent PDDBI & & & \\
$\quad$ Post hoc ANCOVA & 2.407 & .066 & .202 \\
$\quad$ Sensory score & 4.906 & .032 & .107 \\
$\quad$ Maladaptive Behavior (AWP/C) & 10.025 & .003 & .196 \\
$\quad$ Language/Social Abilities (REXCSA) & 1.194 & .281 & .028 \\
$\quad$ Autism Composite score & 9.116 & .004 & .182 \\
Multivariate Sensory and Self-Regulation & & & \\
$\quad$ Post hoc ANCOVA & 5.618 & .002 & .256 \\
$\quad$ Sense Checklist & 12.164 & .001 & .193 \\
$\quad$ Self-Regulation Checklist & 15.626 & .00002 & .235 \\
\hline
\end{tabular}

Note. Pretreatment scores were used as covariates to control for initial difference. $\mathrm{PDDBI}=$ Pervasive Developmental Disorders Behavior Inventory; AWP/C = Approach/Withdrawal Problems Composite; REXSCA = Receptive/Expressive Social Communication Abilities.

aMANCOVA Fs are the Pillai's Trace.

${ }^{\mathrm{b}}$ Partial $\eta^{2}$ as an effect size estimate can be categorized as small (.01-.06), medium (.06-.14), or large (>.14).

\section{Equivalence of Treatment Effects: QST Home Program Versus Control Group}

ANCOVA and MANCOVA were used to determine whether any of the differences in pre-post results shown in Table 3 rose to the level of statistical difference. They did not. In comparing treatment effects between the Dual and Home Programs, none of the differences were significant. Fs ranged from 1.624 to 0.001 with corresponding $p$ values ranging from .207 to .976 (Table 4).

To determine whether the effects were then equivalent, we used a CI approach, which compared a predetermined zone of equivalence to a $95 \% \mathrm{CI}$ around the difference in adjusted mean postassessment scores. We determined the conservative zone of equivalence as being within $+15 \%$ of the postassessment score of the Dual Program intervention, with the mean equal to 0 .

Treatment effects were deemed equivalent when the 95\% CI for the differences in adjusted mean postassessment scores fell wholly within the predetermined zone of equivalence. Treatment effects were deemed indeterminate if the $95 \%$ CI for the differences in adjusted mean postassessment scores extended beyond the zone of equivalence in either direction. Using this analysis, it was determined that the treatment effects were equivalent on PDDBI Sensory Impairment, Maladaptive Behavior, Language and Social Skills, and Autism Composite scores. Results were indeterminate for measures of Autism (ABC), parental stress, and the SSC. These results are also presented in Table 4.

\section{Relationship Between Sensory Regulation Impairment and Autism}

To determine the relationship between sensory regulation impairment and autism, a series of correlation analyses was conducted. First, the correlation between sensory regulation and autism on pretreatment measures was determined for all children in the Dual and Home Program treatments. Correlations were similar for the Dual and Home Program treatments. This was followed by correlations between pretreatment measures of sensory regulation and changes in measures in autism. Correlations were in opposite directions, and the difference in coefficients was statistically significant $(z=2.504)$. Finally, correlations between change scores on measures of sensory regulation and autism were conducted. Again, correlations were similar for the Dual and Home Program treatments.

Results indicate that there might be a relationship between level of sensory regulation impairment at pretreatment and the effectiveness of the Dual and Home Program treatments.

\section{Treatment by Severity-of-Impairment Interaction Effects}

To determine whether the Home and Dual Programs had differential effects depending on the severity of initial parent stress or initial sensory regulation impairment, we ran separate two-way MANOVAs comparing pretest and posttest scores on the PDDBI scales. In both cases, Box's (1949) test for equality of covariance matrices were insignificant.

Parental Stress by Treatment. Treatment type was not significant, $F(4,82)=0.242, p=.914$. Initial Parental Stress was not significant, either, $F(4,82)=0.296, p=$ .880. Similarly, the interaction between treatment type and parental stress was not significant, $F(4,82)=1.63$, $p=.174$.

Post hoc two-way ANOVAs found significant interaction effects between treatment type and level of parental stress for behaviors, $F(1,85)=4.218, p=.043$, and overall autism, $F(1,85)=5.839, p=.018$. In both cases, children with more stressed parents in the Dual Program intervention and less stressed parents in the Home Program intervention experienced significantly greater improvements.

Sensory and Self-Regulation Impairment by Treatment. Treatment type was not significant, $F(4,98)=0.591$, $p=.670$. Sensory and systems impairment was not 


\begin{tabular}{|c|c|c|c|c|c|c|c|c|c|c|c|c|}
\hline \multirow[b]{2}{*}{ Scale } & \multicolumn{6}{|c|}{ QST Home Program Treatment Group } & \multicolumn{6}{|c|}{ QST Dual Program Treatment Group } \\
\hline & $n$ & Preassessment & Postassessment & Change & $p<$ & Cohen's $d$ & $n$ & Preassessment & Postassessment & Change & $p<$ & Cohen's $d$ \\
\hline \multicolumn{13}{|l|}{ Autism Behavior } \\
\hline Checklist & 17 & $76.3(19.6)$ & $56.1(26.4)$ & -20.2 & .001 & 1.03 & 119 & $65.2(27.0)$ & $48.3(26.3)$ & -16.9 & .00001 & 0.63 \\
\hline Parent Stress Index & 39 & $24.0(12.1)$ & 15.0 & -9.0 & .0001 & 0.74 & 71 & $22.3 \quad(9.9)$ & $14.9(7.5)$ & -7.4 & .00001 & 0.75 \\
\hline \multicolumn{13}{|l|}{$\begin{array}{l}\text { Parent PDDBI }(M=50 \\
\quad S D=10)\end{array}$} \\
\hline Sensory score & 29 & $56.4(10.6)$ & $50.1(11.8)$ & -6.3 & .001 & 0.59 & 76 & $54.2(9.8)$ & $47.4 \quad(9.8)$ & -6.8 & .00001 & 0.69 \\
\hline $\begin{array}{l}\text { Maladaptive Behavior } \\
\quad(\mathrm{AWP} / \mathrm{C})\end{array}$ & 29 & $60.9(13.0)$ & $52.3(14.9)$ & -8.6 & .0001 & 0.66 & 76 & $57.9(10.2)$ & $47.7 \quad(9.1)$ & -10.2 & .00001 & 1.0 \\
\hline \multicolumn{13}{|l|}{$\begin{array}{l}\text { Social/Language/ } \\
\text { Communication }\end{array}$} \\
\hline Abilities (REXSCA/C) & 29 & $49.9(11.4)$ & $53.0(10.7)$ & 3.1 & .01 & 0.27 & 76 & $51.5(10.8)$ & $55.8(11.2)$ & 4.3 & .00001 & 0.40 \\
\hline Autism Composite Score & 29 & $59.8(11.1)$ & $50.9(14.8)$ & -8.9 & .0001 & 0.80 & 76 & $55.2(11.2)$ & $44.9(11.6)$ & -10.3 & .00001 & 0.92 \\
\hline $\begin{array}{l}\text { Sense and Self-Regulation } \\
\text { Sense Checklist (0-75) }\end{array}$ & 38 & $38.1(12.1)$ & $28.5(12.2)$ & -9.6 & .001 & 0.79 & 92 & $43.5(15.2)$ & $31.1(13.3)$ & -12.4 & .00001 & 0.82 \\
\hline $\begin{array}{l}\text { Self-Regulation } \\
\text { Checklist (0-99) }\end{array}$ & 38 & $49.1(11.7)$ & $39.2(14.7)$ & -9.9 & .0001 & 0.85 & 92 & $33.2(17.1)$ & $24.3(22.1)$ & -8.9 & .00001 & 0.52 \\
\hline
\end{tabular}

Note. PDDBI = Pervasive Developmental Disorders Behavior Inventory; AWP/C = Approach/Withdrawal Problems Composite; REXSCA/C = Receptive/Expressive Social Communication Abilities Composite.

significant, either, $F(4,98)=0.872, p=.484$. However, the interaction between sensory and systems impairment and treatment type was significant $F(4,98)=3.646$, $p=.008$.

Post hoc two-way ANOVAs found significant interaction effects between treatment type and sensory regulation impairment for behaviors, $F(1,101)=11.53$, $p=.001$; language, $F(1,101)=4.70, p=.032$; and autism, $F(1,101)=8.25, p=.004$. In each case, children with more significant sensory and self-regulation impairment in the Dual Program intervention and less significant sensory and self-regulation impairment in the Home Program intervention experienced significantly greater improvements.

\section{Summary of Results}

Efficacy of the QST Home Program Compared With Wait-List Control Participants. Scores from the ABC, the APSI, the parent PDDBI, and the SSC were used in this analysis. Significant intervention effects were found for the PDDBI, the SSC, and the APSI. The effect sizes (partial $\eta^{2}$ ) were all in the medium to large range.

Table 4. Summary of Analysis of Covariance (ANCOVA) and Multivariate Analysis of Covariance (MANCOVA) Results for Intervention Effects on Measures of Sensory Impairment, Behavior, and Developmental Skills

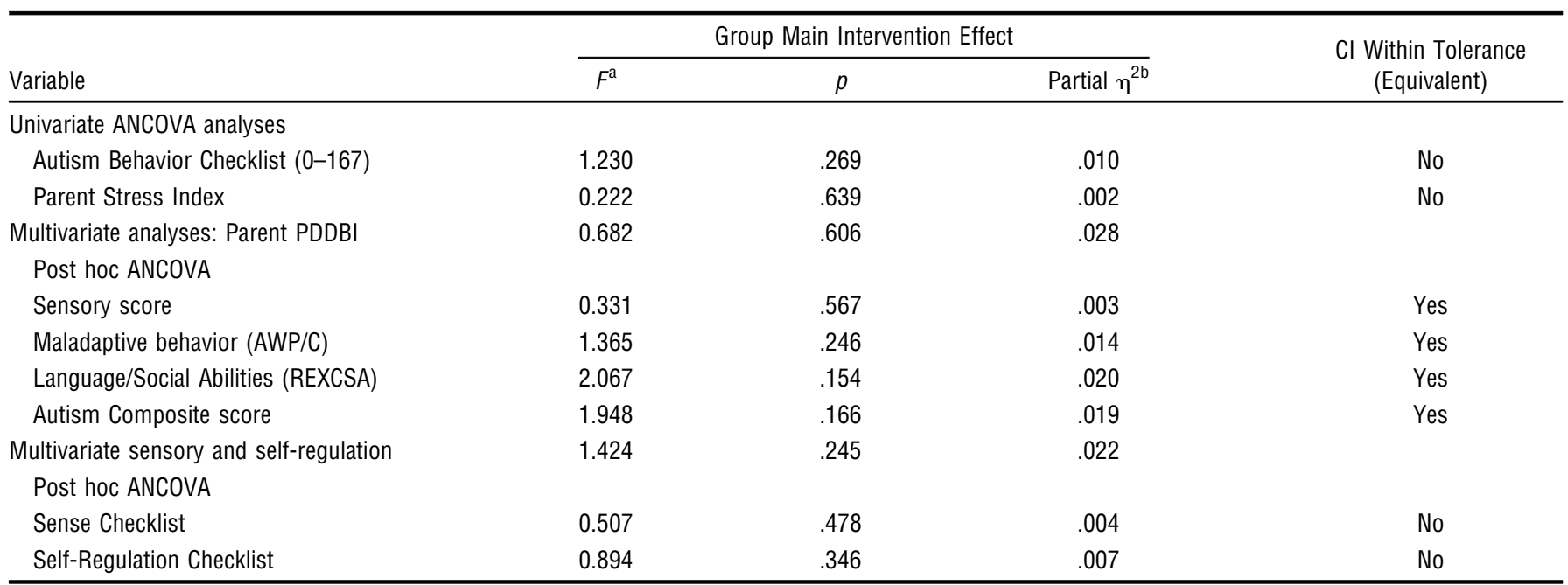

Note. Pretreatment scores used as covariates to control for initial difference. $\mathrm{Cl}=$ confidence interval; $\mathrm{PDDBI}=$ Pervasive Developmental Disorders Behavior Inventory; AWP/C = Approach/Withdrawal Problems Composite; REXSCA/C = Receptive/Expressive Social Communication Abilities Composite.

aMANCOVA Fs are the Pillai's Trace.

${ }^{\mathrm{b}}$ Partial $\eta^{2}$ as an effect size estimate can be categorized as small (.01-.06), medium (.06-.14), or large (>.14). 
Equivalence of the Dual and Home Program Treatments' Effects. No significant differences were found in outcomes from the Dual and Home Program treatments. Moreover, results were found to be equivalent on several outcomes. Results were indeterminate for several outcomes (neither statistically significant nor equivalent). Given the relatively small sample sizes and large variances, these findings are encouraging.

Differential Effects of Dual and Home Program Treatments by Severity of Initial Condition. No main effects were found for either type of treatment or severity of initial condition separately. A main effect was found for the interaction between treatment and severity of sensory and selfregulation impairment on the PDDBI scales. Post hoc analyses indicated interaction effects for both parental stress and initial severity of impairment on several of the PDDBI scales, with more severely impaired participants doing better in the Dual Program treatment and less severely impaired participants doing better in the Home Program treatment.

\section{Discussion}

This pilot study is the most recent in a decade of research collaboration among the Teaching Research Institute, Western Oregon University, and several state-sponsored early intervention programs in Oregon. It originated in response to a request from early intervention program administrators to develop a parent-delivered treatment program that, in contrast to the QST Dual Program, would require less staff support and no direct treatment by staff. In making the request, administrators were aware that this intervention might have less power than the dual intervention and that research has shown that parents are sometimes less successful in delivering a skilled program than trained staff. Nevertheless, they were interested in whether a parent-delivered intervention might be a viable option.

We set out to measure two types of efficacy: (1) Was the training and support program adequate (i.e., were parents able to learn the QST treatment and follow through with it on a daily basis for $4 \mathrm{mo}$ ), and (2) did the intervention result in any measurable differences in the children in the home and preschool setting? If these questions could be answered in the affirmative, then administrators had a third question: Do the results of this pilot study suggest that this intervention can be used before or in place of the more staff-intensive Dual Program intervention and, if so, according to what criteria?

The results show that the training and support program was adequate for parents to learn to give the treatment correctly and to incorporate it into the family's daily routine. Trainers observed parents giving the treatment on seven occasions and reported that it was being done correctly. Evaluation of outcomes in the classroom setting by preschool teachers showed a decrease in autistic behavior with a small effect size. Evaluation of outcomes in the home setting by parents confirmed the improvement reported by teachers. The results of the group as a whole showed that the Home Program had less power than the Dual Program but was nonetheless of benefit both in the school and in the home settings.

It was not surprising that the more severely affected children and their correspondingly more severely stressed parents had significantly better outcomes with the Dual Program than with the Home Program. The Dual Program gave children 20 additional treatments from QST trainers that were not included in the Home Program and, in so doing, gave parents 13 additional support and coaching sessions to develop parent QST skills than were included in the Home Program. With more child treatment and parent coaching directed toward situations of greater severity, outcomes were better.

What was surprising is that less severely affected children (and their correspondingly less stressed parents) had significantly better outcomes with the Home Program than with the Dual Program. We cannot be certain that these results will be replicated in a larger group, because the numbers were small and the variances large by the time we divided our sample size into more and less severe. Nevertheless, we are reasonably certain that what made the difference is that the Home Program was more empowering for parents than the Dual Program by virtue of the fact that it was only the parent who gave the treatment. As such, the parents quickly came to realize that they had the power to make their child better. This was far beyond their expectations. We observed that most parents entered the QST program without particular confidence in the treatment or their ability to give it, but the experience of giving a treatment and seeing their child improve as a result of their efforts was tremendously empowering and confidence building for parents in the Home Program. By contrast, the Dual Program parents did not have this experience; rather, they tended to give the credit for improvement to the trainers and did not as readily understand their critical role in the success of treatment. Whether the children sensed their parents' attitudes and emotions or whether the parents were simply more relaxed and positive as a result of being empowered with a sense of agency and confidence, we feel that the Home Program parents may well have been able to give a more effective treatment.

What we can conclude from this discussion is that a baseline level of parent QST skills was adequately 
transmitted by the Home Program and that it was sufficient to result in good outcomes for children who had less severe autism. This baseline level of skills did not result in equally good outcomes for more severely affected children, and these children required more intense intervention for better outcomes. We also came away with a sense of the importance of the parents' sense of agency and confidence to treatment outcomes.

At issue is the range of severity of autism and the skills needed to treat it with QST. Severity of autism, in our experience, is reflected on the skin of the child in the severity of his or her reactions to touch of the 12 parts of the body encompassed by the 12 QST movements. According to principles of Chinese medicine, these reactions are specifically reflective of the location and degree of impairment. QST is a skill-based tactile therapy that is specifically tuned to the child's tactile responses, and the QST parent and trainer training is rich in strategies to transform these from discomfort and avoidance into pleasure and relaxation. This transformation takes time and skills: The more severe the impairments are, the more time and skills are required on the part of both parent and trainer to navigate the child's difficulties.

In conclusion, perhaps our response to the administrators' third question is that the Home Program can be considered first-line therapy for less severely affected children, and the Dual Program can be reserved as initial treatment of those who are more severely affected. In the meantime, we hope to be able to reach more definitive conclusions by carrying out a larger study exploring the relative effect of the Home and the Dual Programs on both children and parents.

\section{Acknowledgments}

We gratefully acknowledge the families and early intervention programs that participated in our research. In addition, we thank the Curry Stone Foundation and Northwest Health Foundation, whose generous support made this research possible.

\section{References}

Barlow, J. H., Powell, L. A., Gilchrist, M., \& Fotiadou, M. (2008). The effectiveness of the Training and Support Program for parents of children with disabilities: A randomized controlled trial. Journal of Psychosomatic Research, 64, 55-62. doi:10.1016/j.jpsychores.2007. 06.006

Box, G. E. P. (1949). A general distribution theory for a class of likelihood criteria. Biometrika, 36, 317-346.

Cohen, I. L., \& Sudhalter, V. (2005). PDD Behavior Inventory. Lutz, FL: Psychological Assessment Resources.
Cohen, J. (1988). Statistical power analysis for the behavioral sciences (2nd ed.). New York: Academic Press.

Diggle, T., McConachie, H. R., \& Randle, V. R. (2003). Parent-mediated early intervention for young children with autism spectrum disorder. Cochrane Database of Systematic Reviews, 2003. CD003496. doi: 10.1002/ 14651858.CD003496

Eaves, R., \& Williams, T., Jr. (2006). The reliability and construct validity of ratings for the Autism Behavior Checklist. Psychology in the Schools, 43, 129-142. doi:10.1002/ pits. 20122

Haase, R. F. (1983). Classical and partial eta square in multifactor ANOVA designs. Educational and Psychological Measurement, 43, 35-39. doi:10.1177/001316448304300105

Hedges, L. V. (1982). Statistical methodology in meta-analysis. Princeton, NJ: Educational Testing Service.

Interagency Autism Coordinating Committee. (2011). 2011 IACC strategic plan for autism spectrum disorder research. Washington, DC: U.S. Department of Health and $\mathrm{Hu}-$ man Services. Retrieved May 26, 2011, from http://iacc.hhs. gov/strategic-plan/2011/index.shtml

Krug, D., Arick, J., \& Almond, P. (1980). Behavior checklist for identifying severely handicapped individuals with high levels of autistic behavior. Journal of Child Psychology and Psychiatry, and Allied Disciplines, 21, 221-229.

Krug, D., Arick, J., \& Almond, P. (1993). Autism screening instrument for educational planning. Austin, TX: Pro-Ed.

Krug, D., Arick, J., \& Almond, P. (2003). ASIEP-3: Autism Screening Instrument for Educational Planning. Los Angeles: Western Psychological Services.

McConachie, H., \& Diggle, T. (2007). Parent-implemented early intervention for young children with autism spectrum disorder: A systematic review. Journal of Evaluation in Clinical Practice, 13, 120-129. doi:10.1111/j.13652753.2006.00674.x

Mecklin, C. J. (2002, April). The use of equivalence testing in conjunction with standard hypothesis testing and effect sizes. Paper presented at the annual meeting of the American Educational Research Association, New Orleans, LA.

Ospina, M. B., Krebs Seida, J., Clark, B., Karkhaneh, M., Hartling, L., Tjosvold, L., et al. (2008). Behavioural and developmental interventions for autism spectrum disorder: A clinical systematic review. PLoS ONE, 3, e3755. doi: 10.1371/journal.pone.0003755

Schuirmann, D. (1981). On hypothesis testing to determine if the mean of the normal distribution is contained in a known interval. Biometrics, 37, 617.

Schuirmann, D. J. (1987). A comparison of the two one-sided procedure and the power approach for assessing the equivalence of average bioavailability. Journal of Pharmacokinetics and Biopharmaceutics, 15, 657-680. doi: 10.1007/ BF01068419

Silva, L. M. T. (2008). Qigong at home: The Eastern medicine way to help your child. Salem, OR: Qigong Sensory Training Institute.

Silva, L. M. T., Ayres, R., \& Schalock, M. (2008). Outcomes of a pilot training program in a Qigong massage intervention for young children with autism. American Journal of Occupational Therapy, 62, 538-546. 
Silva, L. (2011). Qigong massage for your child with autism: A home program from Chinese medicine. London: Singing Dragon.

Silva, L. M. T., Ayres, R., \& Schalock, M. (2011). A model and treatment for autism at the convergence of Chinese medicine and Western science: First 130 cases. Chinese Journal of Integrative Medicine, 17, 421-429.

Silva, L. M. T., \& Schalock, M. (2011). Autism Parenting Stress Index: Initial psychometric evidence. Journal of Autism and Developmental Disabilities. Advance online publication. doi: 10.1007/s10803-011-1274-1
Silva, L. M. T., \& Schalock, M. (in press). Sense and SelfRegulation Checklist: Initial psychometric evidence and indings. American Journal of Occupational Therapy.

Silva, L. M. T., Schalock, M., Ayres, R., Bunse, C., \& Budden, S. (2009). Qigong massage treatment for sensory and selfregulation problems in young children with autism: A randomized controlled trial. American Journal of Occupational Therapy, 63, 423-432.

Tabachnick, B. G., \& Fidell, L. S. (1989). Using multivariate statistics (2nd ed.). New York: Harper \& Row. 